To appear in Proc. Roy. Soc. Edinb. Sect. A

\title{
HOLOMORPHIC EXTENDIBILITY AND MAPPING DEGREE
}

\author{
Josip Globevnik
}

\begin{abstract}
Let $D$ be a bounded, finitely connected domain in $\mathbb{C}$ without isolated points in the boundary and let $f$ be a continous function on $b D$. Let $\tilde{f}$ be a continuous extension of $f$ to $\bar{D}$. We prove that $f$ extends holomorphically through $D$ if and only if the degree of $\tilde{f}+h$ is nonnegative for every holomorphic function $h$ on $D$ such that $\tilde{f}+h$ is bounded away from zero near $b D$.
\end{abstract}

\section{Introduction and the main result}

H. Alexander and J. Wermer [AW] obtained a characterization of those compact submanifolds of $\mathbb{C}^{N}$ which are boundaries of analytic varieties in terms of their linking numbers with respect to algebraic varieties. Their results inspired some work on characterizing continuous boundary values of holomorphic functions in terms of mapping degree $[\mathrm{S}]$ and, in the case of one variable, in terms of the argument principle [S, G1, G2] which brought some new insights also into the classical one variable theory.

Let $D$ be a bounded domain in $\mathbb{C}$ and let $A(D)$ be the algebra of all continuous functions on $\bar{D}$ which are holomorphic on $D$.

In the special case when $D$ is bounded by finitely many pairwise disjoint simple closed curves the boundary values of functions from $A(D)$ can be characterized in terms of the argument principle:

THEOREM 1.1 [G2] Let $D \subset \mathbb{C}$ be a bounded domain whose boundary consists of a finite number of pairwise disjoint simple closed curves. A continuous function $f$ on $b D$ extends to a function in $A(D)$ if and only if for each $g \in A(D)$ such that $f+g$ has no zero on $b D$, the change of argument of $f+g$ along $b D$ is nonnegative.

We would like to obtain a similar theorem for general domains. Trying to do this we first formulate Theorem 1.1 in terms of mapping degree.

Let $\Psi$ be a continuous function on $b D$ which does not vanish on $b D$. Let $\tilde{\Psi}$ be a continuous extension of $\Psi$ to $\bar{D}$. Approximate $\tilde{\Psi}$ on $\bar{D}$ uniformly by a function $G$ which is smooth in an open neighbourhood of $\bar{D}$ and which does not vanish on $b D$. Perturbing $G$ slightly we may assume that 0 is a regular value of $G$ so that $G^{-1}(0) \cap D$ is a finite subset of $D$ and each point in $G^{-1}(0) \cap D$ is a regular point of $G$. Let $\nu$ be the number of points in $G^{-1}(0) \cap D$ at which $G$ preserves orientation minus the number of points in $G^{-1}(0) \cap D$ at which $G$ reverses orientation. The number $\nu$ depends neither on the choice of the extension $\tilde{\Psi}$ of $\Psi$ nor on the choice of $G$ provided that $G$ approximates $\tilde{\Psi}$ on $\bar{D}$ well enough [D]. We shall call the number $\nu$ the degree of $\Psi, \nu=\operatorname{deg}(\Psi)$. We mention some of its properties. 
If $\Psi_{t}, 0 \leq t \leq 1$, is a continuous family of continuous functions on $b D$ such that $\Psi_{t} \neq 0$ on $b D$ for all $t, 0 \leq t \leq 1$, then $\operatorname{deg}\left(\Psi_{1}\right)=\operatorname{deg}\left(\Psi_{0}\right)$. If $\tilde{\Psi}$ is a continuous function on $\bar{D}$ and $D_{1} \subset D$ is an open set such that $\tilde{\Psi}$ has no zero on $\bar{D} \backslash D_{1}$ then $\operatorname{deg}(\tilde{\Psi} \mid b D)=\operatorname{deg}\left(\tilde{\Psi} \mid b D_{1}\right)$. Further, if $b D$ consists of a finite number of pairwise disjoint simple closed curves then $2 \pi \operatorname{deg}(\Psi)$ equals the change of argument of $\Psi$ along $b D[D]$. In the special case when $\tilde{\Psi}$ is holomorphic on $D$ then $\tilde{\Psi}$ preserves orientation and so the degree of $\Psi$ equals the number of zeros of $\tilde{\Psi}$ in $D$.

We should perhaps point out that usually one calls the number $\nu$ above the degree of $\tilde{\Psi}$ so that one talks about the degree of continuous functions on $\bar{D}$ that have no zero on $b D$. However, since two such functions have the same degree provided that they coincide on $b D[D]$ one, can, as we do, talk about the degree of continuous functions on $b D$ without zeros on $b D$.

One can rewrite Theorem 1.1. as

THEOREM 1.2 Let $D$ be as in Theorem 1.1. A continuous function $f$ extends holomorphically through $D$ if and only if deg $(f+g) \geq 0$ whenever $g \in A(D)$ is such that $f+g$ has no zero on $b D$.

Example [S] Let $\Delta$ be the open unit disc in $\mathbb{C}$ and let $D=\Delta \backslash[0,1)$. Define

$$
f(z)= \begin{cases}z & (z \in b \Delta) \\ 1 & (z \in[0,1))\end{cases}
$$

The function $f$ is continuous on $b D=b \Delta \cup[0,1)$. Note that every $h \in A(D)$ is actually holomorphic on $\Delta$ so that $A(D)=A(\Delta)$. Let $h \in A(D)$ be such that $f+h \neq 0$ on $b D$ and let $G$ be a continuous extension of $f+h \mid b D$ to $\bar{D}=\bar{\Delta}$. Then $\operatorname{deg}(G \mid b D)=$ $\operatorname{deg}\left(G \mid b D_{r}\right)$ where $D_{r}=\Delta \backslash([0,1]+r \bar{\Delta})$ provided that $r>0$ is sufficiently small. The domain $D_{r}$ is bounded by a simple closed curve so the change of argument of $G$ along $b D_{r}$ equals $2 \pi \operatorname{deg}\left(G \mid b D_{r}\right)$. Since $G$ is continuous on $\bar{\Delta}$ the change of argument of $G$ along $\bar{D} \cap b([0,1]+r \Delta)$ tends to zero as $r \searrow 0$ and the change of argument of $G$ along $b D \backslash([0,1]+r \Delta)$ tends to the change of argument of $G$ along $b \Delta$ which, by the argument principle, equals $2 \pi$ times the number of zeros of $z \mapsto z+h(z)$ in $\Delta$. Thus, for any $h \in A(D)$ such that $f+h \neq 0$ on $b D$, the degree of $f+h$ is nonnegative, yet $f$ does not extend holomorphically through $D$. It follows that in general Theorem 1.2 does not hold for more general domains than the ones bounded by finitely many pairwise disjoint simple closed curves.

In the present paper we prove an analogue of Theorem 1.2 for bounded, finitely connected domains in $\mathbb{C}$ without isolated points in the boundary.

Let $D$ be a bounded domain in $\mathbb{C}$. We first define the degree of functions which are continuous and nonzero on $U \cap D$ for some open neighbourhood $U$ of $b D$ in $\mathbb{C}$.

DEFINITION 1.3 Let $U$ be an open neighbourhood of $b D$ in $\mathbb{C}$ and let $\Psi$ be a continuous function on $U \cap D$ which has no zero on $U \cap D$. Let $W$ be a relatively compact open subset of $D$ which contains $D \backslash U$. We define the degree of $\Psi$ as $\operatorname{deg}(\Psi)=\operatorname{deg}(\Psi \mid b W))$.

By the properties of the degree mentioned in Section $1, \operatorname{deg}(\Psi)$ is well defined. It does not depend on the choice of $W$. Moreover, it does not depend on the choice of $U$ : if $U_{1} \subset U$ is 
a neighbourhood of $b D$ in $\mathbb{C}$ then $\operatorname{deg}\left(\Psi \mid U_{1} \cap D\right)=\operatorname{deg}(\Psi)$.

One can express the degree in terms of the change of the $\operatorname{argument.~Exhaust~} D$ by a sequence $D_{m}$ of domains

$$
D_{1} \subset \subset D_{2} \subset \subset \cdots, \cup_{m=1}^{\infty} D_{m}=D
$$

such that for each $m, b D_{m}$ consists of finitely many pairwise disjoint simple closed curves. If $\Psi$ is a continuous function on $U \cap D$ for some open neighbourhood $U$ of $b D$ in $\mathbb{C}$ such that $\Psi \neq 0$ on $U \cap D$ then there is an $m_{0}$ such that $2 \pi \operatorname{deg}(\Psi)$ equals the change of argument of $\Psi$ along $b D_{m}$ for all $m \geq m_{0}$.

DEFINITION 1.4 Let $\Phi$ be a function on $U \cap D$ where $U$ is a neighbourhood of $b D$ in $\mathbb{C}$. We say that $\Phi$ is bounded away from zero near $b D$ if there are a neighbourhood $V \subset U$ of $b D$ and $\delta>0$ such that $|\Phi| \geq \delta$ on $V \cap D$.

Our main result is

THEOREM 1.5 Let $D$ be a bounded, finitely connected domain in $\mathbb{C}$ without isolated points in the boundary and let $f$ be a continous function on $b D$. Let $\tilde{f}$ be a continuous extension of $f$ to $\bar{D}$. The function $f$ extends holomorphically through $D$ if and only if the degree of $\tilde{f}+h$ is nonnegative for every holomorphic function $h$ on $D$ such that $\tilde{f}+h$ is bounded away from zero near bD.

\section{Single valued conjugates}

Let $D$ be as in Theorem 1.5. Exhaust $D$ by a sequence of domains $D_{m}$ as in Section 1. Let $\Gamma_{1}, \Gamma_{2}, \cdots \Gamma_{n}$ be the components of $b D$ such that $\Gamma_{n}$ contains the boundary of the unbounded component of $\mathbb{C} \backslash \bar{D}$. Observe that our conditions imply that $D$ is a Dirichlet domain, that is, for every continuous function $\phi$ on $b D$ there is a continuous extension of $\phi$ to $\bar{D}$ which is harmonic on $D$. This extension is unique and we denote it by $\mathcal{H}(\phi)$.

There are smoothly bounded Jordan domains $\Omega_{j}, 1 \leq j \leq n-1$, such that $\Gamma_{j} \subset$ $\Omega_{j}(1 \leq j \leq n-1)$, such that the closures $\bar{\Omega}_{j}$ are pairwise disjoint and such that $b \Omega_{j} \subset$ $D(1 \leq j \leq n-1)$.

A holomorphic function $h$ on $D$ has a single valued primitive function on $D$ if and only if

$$
\int_{b \Omega_{j}} h(z) d z=0 \quad(1 \leq j \leq n-1)
$$

For each $j, 1 \leq j \leq n-1$, let $\omega_{j}$ be the continuous function on $\bar{D}$ which is harmonic on $D$ and which satisfies $\omega_{j}\left|\Gamma_{j} \equiv 1, \omega_{j}\right| \Gamma_{k} \equiv 0(k \neq j, 1 \leq k \leq n-1)$. We shall need the following lemma which is well known for smoothly bounded domains [B].

LEMMA 2.1 Given a harmonic function $h$ on $D$ there is a unique $(n-1)$-tuple $c_{1}(h)$, $c_{2}(h), \cdots c_{n-1}(h)$ of constants such that $h+\sum_{j=1}^{m-1} c_{j}(h) \omega_{j}$ has a single valued conjugate on $D$.

For each $k, 1 \leq k \leq n-1$, let $F_{k}$ be a multivalued holomorphic function on $D$ whose 
real part is $\omega_{k}$. Then $F_{k}^{\prime}$ is single valued on $D$ and for each $j, 1 \leq j \leq n-1$, the integral

$$
\int_{b \Omega_{j}} F_{k}^{\prime}(z) d z=2 \pi i \alpha_{k, j}
$$

is purely imaginary. We use the reasoning from [E, p.276] to show that the (real) matrix $\left[\alpha_{k, j}\right]$ is nonsingular. Suppose that this is not the case. Then there are real constants $\lambda_{1}, \lambda_{2}, \cdots \lambda_{n-1}$, not all zero, such that

$$
\sum_{k=1}^{n-1} \lambda_{k} \alpha_{k, j}=0 \quad(1 \leq j \leq n-1)
$$

which implies that

$$
\int_{b \Omega_{j}}\left[\sum_{k=1}^{n-1} \lambda_{k} F_{k}(z)\right] d z=0 \quad(1 \leq j \leq n-1)
$$

so the function $F=\sum_{k=1}^{n-1} \lambda_{k} F_{k}$ is a single valued holomorphic function on $D$. Note that the real part $\Re F$ has a continuous extension $\tilde{\Re F}$ to $\bar{D}$ and

$$
\tilde{\Re F}\left|\Gamma_{k} \equiv \lambda_{k}(1 \leq k \leq n-1), \tilde{\Re F}\right| \Gamma_{n} \equiv 0 .
$$

Suppose that $F$ takes in $D$ a value $w_{0}$ that does not belong to the union of vertical lines $i \mathbb{R}, \lambda_{1}+i \mathbb{R}, \lambda_{2}+i \mathbb{R}, \cdots, \lambda_{n-1}+i \mathbb{R}$. Choose $\delta>0$ so small that $w_{0}$ does not belong to the union of vertical strips $(-\delta, \delta)+i \mathbb{R},\left(\lambda_{1}-\delta, \lambda_{1}+\delta\right)+i \mathbb{R}, \cdots\left(\lambda_{n-1}-\delta, \lambda_{n-1}+\delta\right)+i \mathbb{R}$. Let $w_{0}=F\left(z_{0}\right)$ where $z_{0} \in D$ and notice that $m$ can be chosen so large that $z_{0} \in D_{m}$ and that $F\left(b D_{m}\right)$ is contained in the union of the vertical strips above. This implies that the change of argument of $z \mapsto F(z)-w_{0}$ along $b D_{m}$ is zero, contradicting the fact that $F\left(z_{0}\right)=w_{0}$ for $z_{0} \in D_{m}$. Thus $F(D)$ is contained in the union of the vertical lines above and consequently $F$ is a constant which is not possible since at least one of $\lambda_{k}^{\prime} s$ is different from 0 , say, $\lambda_{j} \neq 0$ and $\tilde{\Re F}\left|\Gamma_{j} \equiv \lambda_{j}, \tilde{\Re F}\right| \Gamma_{n} \equiv 0$. The contradiction shows that the matrix $\left[\alpha_{k, j}\right]$ is nonsingular.

Proof of Lemma 2.1. It suffices to prove that given a real harmonic function $h$ on $D$ there are unique real constants $c_{1}(h), \cdots, c_{n-1}(h)$ such that $h+\sum_{j=1}^{n-1} c_{j}(h) \omega_{j}$ has a single valued conjugate. This is the same as to say that there are unique real constants $c_{1}(h), \cdots, c_{n-1}(h)$ such that $H+\sum_{j=1}^{n-1} c_{j}(h) F_{j}$ is single valued where $H$ is a multiple valued holomorphic function on $D$ whose real part is $h$. This happens if and only if

$$
\int_{b \Omega_{j}}\left[H^{\prime}(z)+\sum_{k=1}^{n-1} c_{k}(h) F_{k}^{\prime}(z)\right] d z=0 \quad(1 \leq j \leq n-1)
$$

so

$$
\sum_{k=1}^{n-1} c_{k}(h)\left[\frac{1}{2 \pi i} \int_{b \Omega_{j}} F_{k}^{\prime}(z) d z\right]=-\frac{1}{2 \pi i} \int_{b \Omega_{j}} H^{\prime}(z) d z \quad(1 \leq j \leq n-1) .
$$


Since the matrix $\left[\alpha_{j, k}\right]$ is nonsingular there is a unique $(n-1)$-tuple $c_{1}(h), \cdots, c_{n-1}(h)$ of real constants such that (2.1) is satisfied. This completes the proof.

\section{Proof of Theorem 1.5}

Observe first that if $U$ is a neighbourhood of $b D$ in $\mathbb{C}$ and $\Phi, \Psi$ are continuous functions on $U \cap \bar{D}$ such that $\Phi \equiv \Psi$ on $b D$, and $h$ is a continuous function on $U \cap D$ such that $\Phi+h$ is bounded away from 0 near $b D$, then $\Psi+h$ is bounded away from 0 near $b D$ and $\operatorname{deg}(\Phi+h)=\operatorname{deg}(\Psi+h)$. To see this notice first that by the assumption there are a neighbourhood $V \subset U$ of $b D$ and $\delta>0$ such that $|\Phi+h| \geq \delta$ on $V \cap D$. Since $\Phi \equiv \Psi$ on $b D$ and since $\Phi$ and $\Psi$ are continuous there is a neighbourhood $V_{1} \subset V$ of $b D$ in $\mathbb{C}$ such that $|\Phi-\Psi|<\delta / 2$ on $V_{1} \cap D$. For each $t, 0 \leq t \leq 1$, we have $|\Phi+t(\Psi-\Phi)+h| \geq|\Phi+h|-\delta / 2 \geq \delta / 2$ on $V_{1}$, that is, for each $t, 0 \leq t \leq 1$, the function $(1-t)(\Phi+h)+t(\Psi+h)$ is bounded away from 0 on $D \cap V_{1}$. In particular, $\Psi+h$ is bounded away from 0 on $D \cap V_{1}$. Moreover, since $(\Psi+h) \mid V_{1}$ is homotopic to $(\Phi+h) \mid V_{1}$ through a family of maps with ranges contained in $\mathbb{C} \backslash\{0\}$ it follows that $\operatorname{deg}(\Psi+h)=\operatorname{deg}(\Phi+h)$.

We now turn to the proof of Theorem 1.5. Denote by $Z$ the identity, $Z(z) \equiv z$. Suppose that $f$ is a continuous function on $b D$ which has a continuous extension $g$ to $\bar{D}$ which is holomorphic on $D$. Let $h$ be a holomorphic function on $D$ such that $g+h$ is bounded away from zero near $b D$. This means that there are an open neighbourhood $U$ of $b D$ in $\mathbb{C}$ and a $\delta>0$ such that $|g+h| \geq \delta>0$ on $U \cap D$. Choose $m$ so large that $b D_{m} \subset U$. Then $2 \pi \operatorname{deg}(g+h)$ equals the change of argument of $g+h$ along $b D_{m}$. Since $g$ and $h$ are holomorphic on $D$ the argument principle implies that the change of argument of $g+h$ along $b D_{m}$ equals $2 \pi$ times the number of zeros of $g+h$ in $D_{m}$ so it is nonnegative. Thus $\operatorname{deg}(g+h) \geq 0$. The resoning above implies that $\tilde{f}+h$ is bounded away from zero near $b D$ and that $\operatorname{deg}(\tilde{f}+h)=\operatorname{deg}(g+h) \geq 0$. This proves the only if part of the theorem.

The proof of the if part is very short in the special case when $D$ is simply connected. It uses the basic fact that a continuous function $f$ on $b D$ extends holomorphically through $D$ if and only if the harmonic extension of $Z f$ equals $z$ times the harmonic extension of $f$, that is, if and only if $\mathcal{H}(Z f)(z) \equiv z \mathcal{H}(f)(z) \quad(z \in D)$ [G2]. So suppose that $f$ does not extend holomorphically through $D$. Then there is an $a \in D$ such that $\mathcal{H}((Z-a) f)(a) \neq 0$, and multiplying $f$ by $e^{i \gamma}, \gamma \in \mathbb{R}$, we may assume that $\mathcal{H}((Z-a) f)(a)=\alpha$ where $\alpha>0$. Since $D$ is simply connected it follows that $\mathcal{H}((Z-a) f)(z)-\alpha=(z-a) F(z)-\overline{(z-a) G(z)}$ where $F$ and $G$ are holomorphic on $D$ and so $\mathcal{H}((Z-a) f)(z)-(z-a) F(z)-(z-a) G(z) \in$ $\alpha+i \mathbb{R}(z \in D)$ which implies that $z \mapsto(1 /(z-a)) \mathcal{H}((Z-a) f)(z)-F(z)-G(z)$ is bounded away from zero near $b D$ and its degree equals -1 .

The proof of the if part in the case when $D$ is multiply connected is more complicated because in general, conjugate functions are no more single valued. We shall use the reasoning from [G2]. Assume that $f$ is a continuous function on $b D$ that does not extend holomorphically through $D$. Denote by $Z$ the identity, $Z(z) \equiv z$ and define

$$
A(a, f)=\mathcal{H}((Z-a) f)(a)=\mathcal{H}(Z f)(a)-a \mathcal{H}(f)(a) \quad(a \in \bar{D})
$$

Since $\mathcal{H}(f)$ is not holomorphic on $D$ it follows that $\{a \in D: A(a, f)=0\}$ is a closed, 
nowhere dense subset of $D$ [G2]. There are constants $c_{k}, d_{k}, 1 \leq k \leq n-1$, such that

$$
\begin{gathered}
\mathcal{H}(f)(z)+\sum_{k=1}^{n-1} c_{k} \omega_{k}(z)=P(z)+\overline{Q(z)} \quad(z \in D) \\
\mathcal{H}(Z f)(z)+\sum_{k=1}^{n-1} d_{k} \omega_{k}(z)=R(z)+\overline{S(z)} \quad(z \in D)
\end{gathered}
$$

where $P, Q, R, S$ are holomorphic functions on $D$. Define

$$
\Phi_{a}(z)=\sum_{j=1}^{n-1}\left[d_{j}-a c_{j}\right] \cdot\left[\omega_{j}(z)-\omega_{j}(a)\right]-A(a, f) .
$$

For each $a \in D$ the function $\Phi_{a}$ is continuous on $\bar{D}$ and harmonic on $D$ and by the preceding discussion, the function $z \mapsto \mathcal{H}((Z-a) f)(z)+\Phi_{a}(z) \quad(z \in D)$ vanishes at $a$ and has a conjugate on $D$, that is, it is of the form $(z-a) F_{a}(z)+\overline{(z-a) G_{a}(z)}$ where $F_{a}$ and $G_{a}$ are single valued holomorphic functions on $D$.

The function $\Phi_{a}$ is constant on each $\Gamma_{k}, 1 \leq k \leq n$. Repeating the proof of Lemma 6.1 in [G2] we see that there is an $a \in D$ such that all these constants are different from 0 , that is, $\Phi_{a}(z) \neq 0(z \in b D)$. Replacing $f$ by $e^{i \gamma} f, \gamma \in \mathbb{R}$ will multiply all these constants with $e^{i \gamma}$ so we may assume with no loss of generality that the real parts of all these constants are different from zero, that is, $\Re \Phi_{a}(z) \neq 0(z \in b D)$. Hence, if

$$
W(z)=\mathcal{H}((Z-a) f)(z)-(z-a) F_{a}(z)-(z-a) G_{a}(z) \quad(z \in D)
$$

it follows that

$$
\Re W(z)=-\Re \Phi_{a}(z) \quad(z \in D)
$$

where the function on the right is continuous on $\bar{D}$ and for each $j, 1 \leq j \leq n$, equal to a nonzero real constant $\beta_{j}$ on $\Gamma_{j}$. This shows that $W$ is bounded away from zero on $U \cap D$ for a neighbourhood $U$ of $b D$. Choose $\delta>0$ so small that $0 \notin \cup_{j=1}^{n}\left[\beta_{j}-\delta, \beta_{j}+\delta\right]$. There is an $m_{0} \in \mathbb{N}$ such that for all $m \geq m_{0}$, we have $W\left(b D_{m}\right) \subset \cup_{j=1}^{n}\left[\beta_{j}-\delta, \beta_{k}+\delta\right]+i \mathbb{R}$. It follows that the change of argument of $W$ along $b D_{m}$ is zero, and consequently, provided that $a \in D_{m_{0}}$, the change of argument of $z \mapsto W(z) /(z-a)$ along $b D_{m}$ equals $-2 \pi$ for all $m \geq m_{0}$. This implies that the function

$$
z \mapsto \frac{1}{z-a} W(z)=\frac{1}{z-a} \mathcal{H}((Z-a) f)(z)-F_{a}(z)-G_{a}(z)
$$

is bounded away from zero near $b D$ and its degree equals -1 . Since the function $z \mapsto$ $(1 /(z-a)) \mathcal{H}((Z-a) f)(z)$ is a continuous extension of $f$ to $\bar{D} \backslash\{0\}$ Proposition 3.1 implies that $\tilde{f}-F_{a}-G_{a}$ is bounded away from zero near $b D$ and $\operatorname{deg}\left(\tilde{f}-F_{a}-G_{a}\right)=-1$. This completes the proof.

\section{An example}


Let $\Delta$ be the open unit disc in $\mathbb{C}$ and let $D=\Delta \backslash\{0\}$. Define

$$
f(z)= \begin{cases}0 & (z \in b \Delta) \\ 1 & (z=0) .\end{cases}
$$

Suppose that $\tilde{f}$ is a continuous extension of $f$ to $\bar{D}=\bar{\Delta}$ and let $h$ be a holomorphic function on $D$ such that $\tilde{f}+h$ is bounded away from 0 on $b D$. In particular, there are $\delta>0$, a neighbourhood $U$ of $b \Delta$ in $\mathbb{C}$ and a neighbourhood $V$ of 0 such that $|\tilde{f}+h| \geq 2 \delta$ on $U \cap \Delta$ and on $V \backslash\{0\}$. Shrinking $U$ and $V$ if necessary we may assume that

$$
\begin{gathered}
|h| \geq \delta \quad \text { on } U \cap \Delta \\
|h+1| \geq \delta \text { on } V \backslash\{0\} .
\end{gathered}
$$

We have $2 \pi \operatorname{deg}(\tilde{f}+h)=V_{R}-V_{\rho}$ where $V_{R}$ is the change of argument of $\tilde{f}+h$ along $b(R \Delta)$ for $R<1$ very close to 1 and $V_{\rho}$ is the change of argument of $\tilde{f}+h$ along $b(\rho \Delta)$ for $\rho>0$ very small. Shrinking $U$ if necessary we may assume that $|\tilde{f}|<\delta / 2$ on $U \cap \Delta$. Thus, if $R<1$ is sufficiently close to $1, t \mapsto(h+t \tilde{f}) \mid b(R \Delta)(0 \leq t \leq 1)$ is a homotopy between $h \mid b(R \Delta)$ and $(h+\tilde{f}) \mid b(R \Delta)$ in $\mathbb{C} \backslash\{0\}$ so $V_{R}$ equals the change of argument of $h$ along $b(R \Delta)$. Similarly, shrinking $V$ if necessary we may assume that $|\tilde{f}-1|<\delta / 2$ on $V \backslash\{0\}$, so, provided that $\rho>0$ is sufficiently small, $t \mapsto[h+1+t(\tilde{f}-1)] \mid b(\rho \Delta)(0 \leq t \leq 1)$ is a homotopy between $(h+1) \mid b(\rho \Delta)$ and $[h+\tilde{f}] \mid b(\rho \Delta)$ in $\mathbb{C} \backslash\{0\}$ so $V_{\rho}$ equals the change of argument of $h+1$ along $b(\rho \Delta)$. The condition (4.2) implies that $h$ is either holomorphic at 0 or has a pole at 0 . In the first case the change of argument of $h+1$ along $b(\rho \Delta)$ is zero since $h+1$ has no zero on $\rho \bar{\Delta}$. In the second case there is a $\rho>0$ such that $h$ is large on $b(\rho \Delta)$, say, $|h|>10$. This implies that the change of argument of $h+1$ along $b(\rho \Delta)$ equals the change of argument of $h$ along $b(\rho \Delta)$. Thus, in the first case, $V_{\rho}=0$ and so $V_{R}-V_{\rho}$ equals the number of zeros of $h$ on $R \Delta$ and so is nonnegative. In the second case $V_{R}-V_{\rho}$ equals the number of zeros of $h$ on $R D \backslash \rho \bar{D}$ and so is nonnegative. Thus, $\operatorname{deg}(f+h) \geq 0$ for every holomorphic function $h$ on $D$ such that $f+h$ is bounded away from 0 on $b D$, yet $f$ does not extend holomorphically through $D$. This shows that in Theorem 1.3 the assumption that all components of $b D$ be nondegenerate cannot be omitted.

This work was supported in part by the Ministry of Higher Education, Science and Technology of Slovenia through the research program Analysis and Geometry, Contract No. P1-0291. 


\section{REFERENCES}

[AW] H. Alexander and J. Wermer: Linking numbers and boundaries of varieties. Ann. Math. 151 (2000) 125-150

[B] S. Bell: The Cauchy transform, Potential Theory, and Conformal Mapping. CRC Press, Boca Raton, 1992

[D] K. Deimling: Nonlinear functional analysis. Springer Verlag, Berlin, 1980

[E] M. A. Evgrafov: Analytic functions. Saunders, Philadelphia and London, 1966

[G1] J. Globevnik: Holomorphic extendibility and the argument principle.

Complex Analysis and Dynamical Systems II. Contemp. Math. 382 (2005) 171-175

[G2] J. Globevnik: The argument principle and holomorphic extendibility.

Journ. d'Analyse. Math. 94 (2004) 385-395

[S] E.L.Stout: Boundary values and mapping degree.

Michig. Math. J. 47 (2000) 353-368

[T] M. Tsuji: Potential theory in modern function theory. Maruzen, Tokyo, 1959

Institute of Mathematics, Physics and Mechanics University of Ljubljana, Ljubljana, Slovenia

josip.globevnik@fmf.uni-lj.si 